\title{
Decoding Social Influence and the Wisdom of the Crowd in Financial Trading Network
}

\author{
Wei Pan \\ MIT Media Lab \\ Cambridge, MA 02139 \\ Email:panwei@media.mit.edu
}

\author{
Yaniv Altshuler \\ MIT Media Lab \\ Cambridge, MA 02139 \\ Email: yanival@mit.edu
}

\author{
Alex (Sandy) Pentland \\ MIT Media Lab \\ Cambridge, MA 02139 \\ Email: pentland@mit.edu
}

\begin{abstract}
In this paper, we study roles of social mechanisms in a financial system. Our data come from a novel on-line foreign exchange trading brokerage for individual investors, which also allows investors to form social network ties between each other and copy others' trades. From the dataset, we analyze the dynamics of this connected social influence systems in decision making processes. We discover that generally social trades outperform individual trades, but the social reputation of the top traders is not completely determined by their performance due to social feedback even when users are betting their own money. We also find that social influence plays a significant role in users' trades, especially decisions during periods of uncertainty. We report evidences suggesting that the dynamics of social influence contribute to market overreaction.
\end{abstract}

\section{INTRODUCTION}

Recently the interests on social influence and social dynamics are growing with the rise of Computational Social Science [1]. We see increasing research efforts in measuring and understanding different social dynamics systems: online systems like Twitter, and real living systems ranging from discussion groups [2], a dormitory community [3] to a New England town [4], to name a few.

One particularly interesting type of social systems are the financial systems. Though financial systems have been analyzed dominantly with physics stochastic calculus (i.e. random walk) models such as Black-Sholes [5], they are with no doubt driven by the collective behavior of humans. Key aspects of social science, such as the existence of social influence and the topology restrictions on information flow, are rarely discussed by finance researchers [6]. By adopting social theories into the analysis of different financial systems, this approach may be able to better explain the many mysterious phenomena in the markets, such as overractions and market crash.

On the other side, financial systems are among the best quantitatively documented systems, with datasets of transactions in mill-second resolution. Though many of the data are still only about trades rather than networks, researchers are able to infer the network properties of financial systems with newly developed tools [7] [8] to understand the underlying connectivity from individual trades. In addition, new financial data with explicit social relationships are also becoming available [6].

In this work, we demonstrate how the study of financial systems, from the Computational Social Science point of view, can lead to interesting results for some general social science questions. We also demonstrate that the understanding of financial systems with social science perspectives can be turned into profitable hedging and trading strategies.

Our work is based on a novel retail online brokerage known as "eToro" (http://www.etoro.com). eToro provides users easy tools to trade in foreign exchange and commodity markets. The most important feature of eToro is that users can also look at other users and follow others' trades. We will discuss the detail of our eToro dataset in Section II. Based on this dataset, we will demonstrate how two fundamental challenges in social science are also the fundamental challenges inside the eToro financial system:

The first challenge is whether we can elicit optimal decisions from the wisdom of the crowd. From the literature one may expect that financial systems are among the best crowd wisdom systems, as researchers believe that real cash incentive is the driving force behind optimal rational crowd wisdom [9] [10] [11]. We analyze the performance of the crowd of eToro users in predicting experts( i.e. those who will make the most money in the future), and we discuss the potential causes of our observations. Our results suggest that generally the crowd does much better than individuals. Still, the eToro crowd is subject to social influence dynamics rather than complete rational thinking even under strong monetary incentives. The detail is in Section III.

The second challenge is to understand how social influence alters the dynamics of the crowd. Many social science researchers have studied social influence dynamics on networks [7] [12], and naturally we expect such social influence dynamics to exist in financial systems. With the eToro data, we are able to closely examine how social influence changes decision making process. We discover strong evidence that social influence stimulates speculations and overreactions in financial markets. The results are shown in Section IV.

Readers may argue that eToro is a special type of financial markets, and our results may not be representative. We believe that in the real financial world while market participants can usually trade with any other participant in open platforms such as NYSE, information flow, opinions and influence from other peers, and the eventual trading decisions are often largely constrained by the network connections of traders in a manner similar to the eToro user network. 


\section{DATASETS}

Our data come from eToro (See http://www.etoro.com), an on line discounted retail broker for foreign exchanges and commodities tradings with easy-to-use buying and shortselling mechanisms as well as leverage up to 400 times. In other words, eToro makes trading accessible and fun, as it allows any user to take both long and short positions, with a minimal bid of a few dollars. eToro also magnifies risks in trades, as it allows user to be leveraged. As a result sometimes traders do lose more than $100 \%$ of their position value in a single transaction.

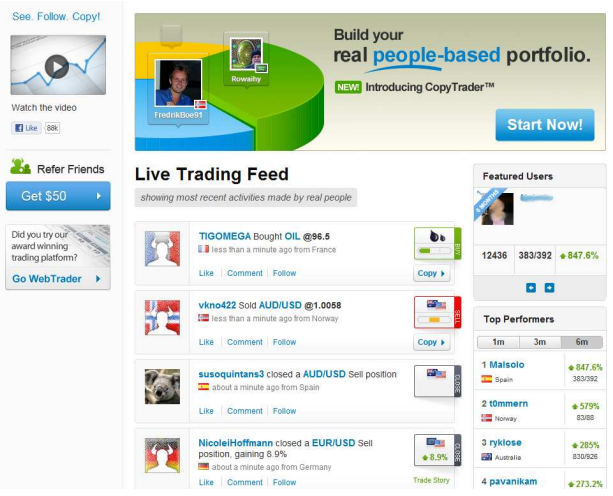

(a) Social Trading Landing Page

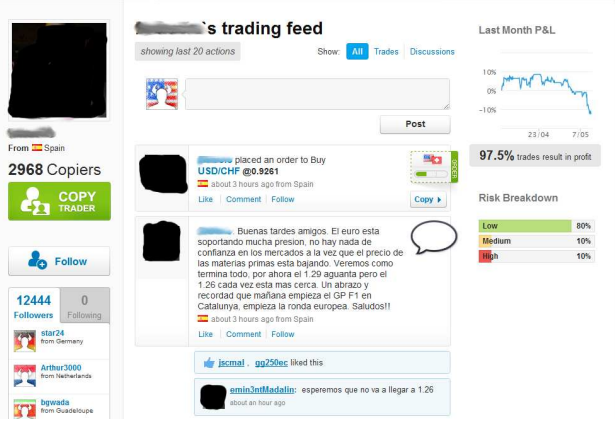

(b) Public Profile for a User

Fig. 1. We show the screenshots for the eToro social trading platform here: a) The general landing page showing the current trades by other users and top ranked traders. Users can click any trade to copy; b) A public profile page for a eToro user (images and names removed), which contains his current trades, messages and most importantly the number of followers mirroring his trades.

However, among all new features, the most interesting feature of eToro is that it provides a social network platform (known as The Open Book) for all traders. We illustrate the main social trading interface in Fig. 1. Users can easily look up other users' trades, portfolios, and past performance. Users can place three types of trades in eToro.

- Single trade: Users can place a normal trade by themselves.

- Copy trade: This mechanism allows a user to place a trade exactly as another user's one single trade. In the following discussion, we will refer to this type of copying as "copy trade". As shown in Fig. 1(a), users can review all current real-time trades, and choose any one to copy.
- Mirror trade: This mechanism allows a user to pick an example user. For every trade the example user makes, eToro automatically executes the same trade on behalf of the user. In the following discussion, we will refer to this type of copying as "mirror trade". Fig. 1(b) shows a user's profile page, where other users can follow and mirror all the trades of this user in his profile page. The word "follow" and "mirror" in the following content is interchangeable.

Our data are composed of over 5.8 million trades from August 2010 to January 2012 (social trading features were launched at early 2011). Among them 3 million trades are non-social independent trades, and around 2.7 million trades are automatically executed "mirror trades" for each user by copying trades from the people the user follows. The rest are "copy trades" that users manually copied from other users' trades.

Like many other crowd-sourcing tasks, the truth is in the hands of the few. Overall most investors in eToro are losing their investments. Though we have to recognize that this period is the most difficult time for our financial systems, it should be noted that eToro provides both long and short mechanisms. Therefore, many successful traders can still profit from shorting the market and other market neutral strategies. We first look at some statistics of all transactions: on average each trade lost $2.8 \%$ in its position size, with $\sigma=40 \%$. The overall total transaction in our datasets is over 47 millions USD, and the total loss from users is around 2 millions USD. User-wise, of all the users who have traded at least once, only $16 \%$ users were able to make a profit during the time period in the dataset. This matches the common belief that in crowd systems there usually exists a very small portion of elites [13].

Nevertheless, the promise in eToro lies in its social features: In Fig. 2, we plot average daily ROI (Return On Investment) of EUR/USD on all the trades of different categories. We find that different types of trades lead to different levels of returns (ANOVA $p<1 e-10$ ), and mirror trades actually generate a positive return ( $t$-test, $p<0.005)$. At a first look, following other users in the crowd seems to be a simple way to make money, and social trades do outperform single trades. In this paper, we will take a deep look into the crowd wisdom to decode how social mechanisms alter crowd behaviors in the eToro financial system.

In the following content, we only focus on one single security, EUR/USD, which is the most liquid instrument on eToro and counts for over $82 \%$ of all transactions on eToro.

\section{ELICITING CROWD WISDOM FOR BEST TRADERS}

While people do recognize the power of human computing in certain tasks that machines are simply not able to do, it is still unclear if a crowd can provide better predictions and decisions about future events [10]. Many researchers defend crowd prediction by suggesting that real money incentives are important, and urge governments to allow real cash flow in prediction market [9]. Financial systems provide the exact 


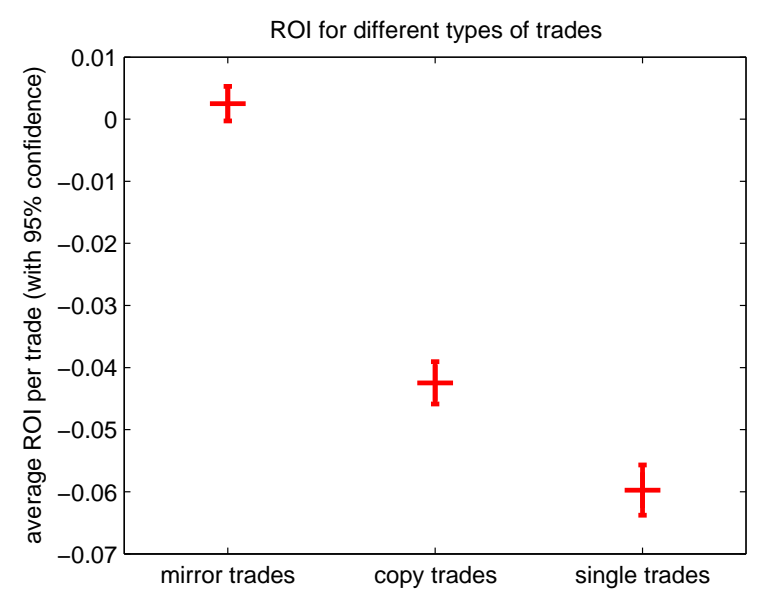

Fig. 2. The mean ROI for all trades of the three types. The returns are significantly different from each other (ANOVA $p<1 e-10$ ), and mirror trades generate significant positive return $(t$-test, $p<0.005)$.

unique perspective in understanding the crowd wisdom, as real money incentives are always involved.

In eToro, one of the best strategies for users is actually to mirror other traders' strategies as seen in Fig. 2. User A can choose to mirror a more sophisticated user B, and eToro will automatically copy and execute user B's all future trades on behalf of user A. Naturally this mirroring behavior implies strong trust in user B from user A, and users who are mirrored and followed the most are likely to be the best traders. We observe in Fig. 2 that mirroring actually generates profit. However, we still would like to know how well the crowd is performing in selecting its leaders to follow.

We study this problem by constructing trading strategies using the wisdom of the crowd. We here construct two portfolio strategies rebalanced daily. The first strategy, referred as the Simple Best strategy, is constructed by looking at the top $t$ users on eToro platforms. On each day, we rank each user by their accumulated continuously compounded return up to this day, and execute the same trades of the top $t$ users by evenly dividing possessed capital among these users in this day. If none of the top $t$ users is trading on a particular day, we don't trade either. We construct the second social strategy by analogy to the first Simple Best Strategy. Everything remains the same except we rank users by their numbers of followers rather than their return performance. The intuition behind this strategy is that the best users recognized by the crowd are the best users on the platform. We execute the same trades of the top $t$ mirrored users by evenly dividing capitals. We refer to this strategy as the Social Best strategy.

In Fig.3, we plot the mean return and Sharpe Ratio for both the Simple Best and Social Best Strategies. Sharpe ratio is defined as the mean return divided by the risk (i.e. variance of the return), which is a common financial metric for riskadjusted return [14]. It seems that the crowd did select a reasonable set of trusted traders to follow, as their return is significantly above average. Also, for both the top 5 and
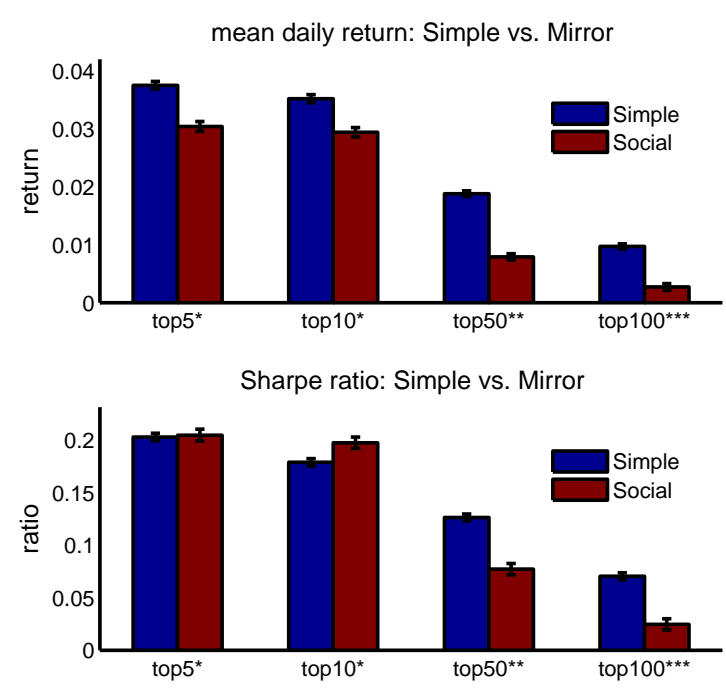

Fig. 3. The mean daily ROI and Sharpe Ratio for both the Simple Best strategy and the Social Best strategy. (*: Both returns are significantly above zero in t-test with $p<0.01$; **: only the Simple Best strategy return is significantly above zero; ***: neither strategy is significantly above zero with $p>0.1$.)

the top 10 strategies, we notice that the performance of the crowd and the performance of the simple best strategy are almost equivalent. However, the Sharpe ratio of the crowd is slightly higher. We suspect that this is due to the fact that crowds are more risk averse. However, when we extend our portfolio to top 50 users using both strategies, we notice that the performance of the crowd drops significantly.

Fig. 3 provides a lot of information: it seems that it is possible to profit from the top users, but there are very few of them. Increasing the diversity of the selected users beyond top 10 will immediately reduces profitability. Another interesting observation is that the crowd performs really well in selecting its top leaders, but fails to outperform a simple algorithm when being asked to select more experts.

To answer this problem, we also plot from the top $20 \%$ users the distributions on their accumulated returns and their numbers of followers in Fig. 4(a) and Fig. 4(b) respectively. It is clear that for performance, very few users exhibit strong trading skills, and the distribution suggests an exponential decaying distribution. However, the number of followers follow a power-law long tail distribution, and unlike trading skills, the distribution is much flatter.

\section{A. Discussion}

Our results match very well with a well-known cultural market study [15], in which the crowd votes for the best songs on an artificial online music sharing website where previous user votes are recorded and displayed. The sharing website has multiple independent universes to attend for repeat studies. Researchers discovered that the "best" songs always rank at the top, but most songs had uncertain ranking outcomes in different universes. In eToro, the top 10 best traders recognized 


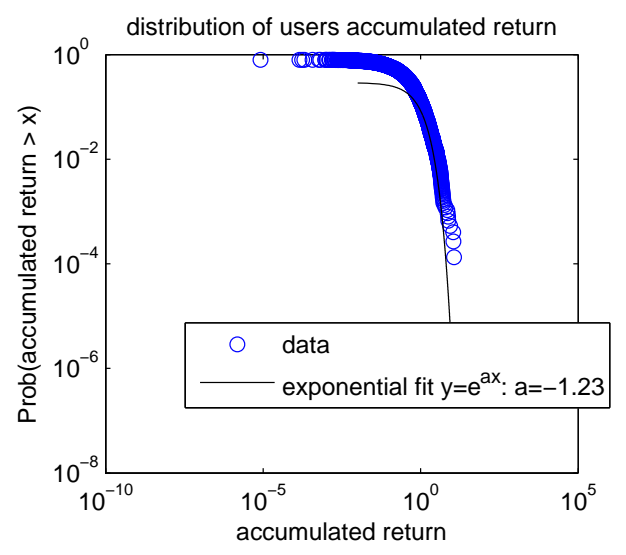

(a) Distribution of Return

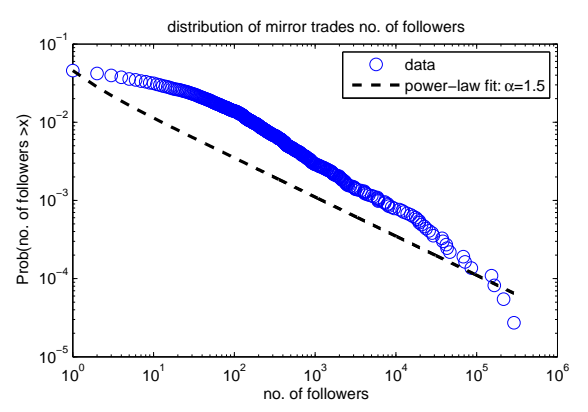

(b) Distribution of Number of Followers

Fig. 4. From the top $20 \%$ users the distributions on both (a) the accumulated return and by (b) the number of followers are plotted here. The distribution of followers show strong power-law pattern, but the accumulated return follows an exponential decay.

by accumulated performance and crowd choices generate comparable return. However, when we further diversify our portfolio by including more top followed users, we start to realize that the crowd's selected experts have less certain skills, just like many top ranked songs in the cultural market study.

We suspect that the process of expert eliciting on eToro is similar to a preferential attachment model [16], because in eToro users are also provided with the information of the number of followers about another user. We notice that the number of followers in Fig. 4(b) forms a distribution close to a power-law curve rather than the exponential distribution of user performance in Fig. 4(a), which is a strong indicator for preference attachment behavior [16]. In other words, users are heavily affected by the existing number of followers when making mirroring decisions, even when eToro completely discloses trading performance and ranks. Under a fully rational model, users' preference should always match the traders' performance. However, we examine the performance of the top 300 most followed users in the system (Fig. 5), and we find that ranking doesn't correlate with the performance. Our finding contradicts the common view for collective intelligence researchers who argue that strong monetary incentives induce more rational decisions [11], and we argue that social feedback does sometimes overweight individual rational thinking. We believe the reason lies in the fundamental basis of the evolutionary human cognitive structure [17].

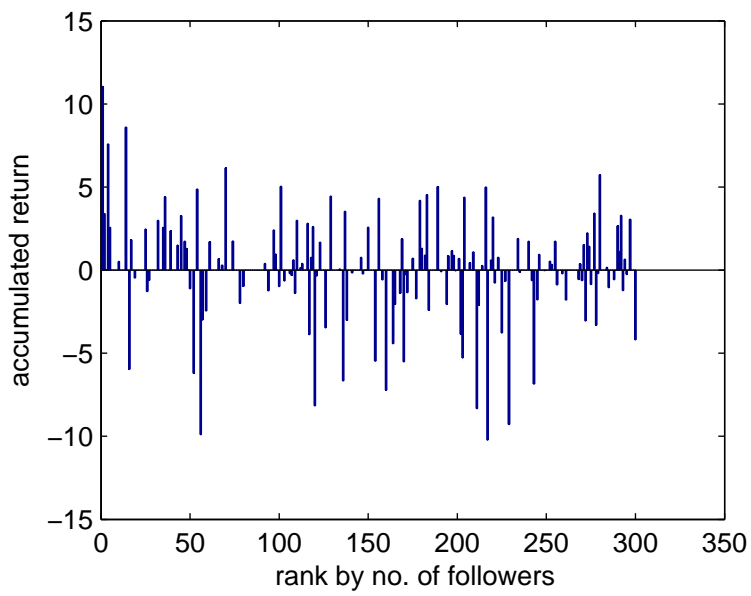

Fig. 5. We plot the accumulated return for the top 300 most followed users in eToro. Except for the top ones, most users' performance seems to be irrelevant of their ranking.

\section{Social Influence in Financial Decision Making}

Besides mirroring other users, eToro also allows users to copy a single trade rather than every trade from another user (the copy trade type). Users can log into the Open Book to look for other users' trades, and click to copy one trade. If a user copies a trade, it implies that the user has come to the Open Book and consulted other users' trades. He/she made the decision based on his/her own judgment as well as the social influence from others. It should be mentioned that copying trades are less common ( $\approx 2 \%$ of all transactions) in eToro.

There is a long line of research studying the effects on social influence in different domains, such as health [4], purchase behavior [18] [19], to name a few. eToro datasets provide us an unique opportunity to unveil social influence in financial trading behavior, and our paper is the first one on this topic as far as we know. Most of the time researchers are unable to know exactly what information and influence a trader receives before making trades, while in eToro we know that all copy trades are clearly under social influence.

We define the following notation: for each day $d$, we compute the percentage of long trades among all single trades denoted as $s_{d}$, and the percentage of long trades among all copy trades as $c_{d}$. We also refer to $s_{d}$ as single market perspective, and $c_{d}$ as crowd market perspective.

We here plot $s_{d}$ and $c_{d}$ in Fig. 6. Our discovery is surprising: while individual beliefs in buys and sells are fairly stable, the speculations in copy trades are a magnitude more volatile $\left(\sigma^{2}\right.$ : 0.006 vs. 0.03 , F-test $p<10^{-36}$ ). Therefore with explicit social inputs, users tend to become more extreme rather than converge. Social influence seems to play an important role in driving market volatility.

We plot $\left(s_{d}, c_{d}\right), \forall d$ on a 2 -d space, and the results are illustrated in Fig. 7. Fig. 7 clearly suggests that a linear correlation with slope $=1.37$. In other words, the explicit exposure 


\section{daily percentage of long and short}

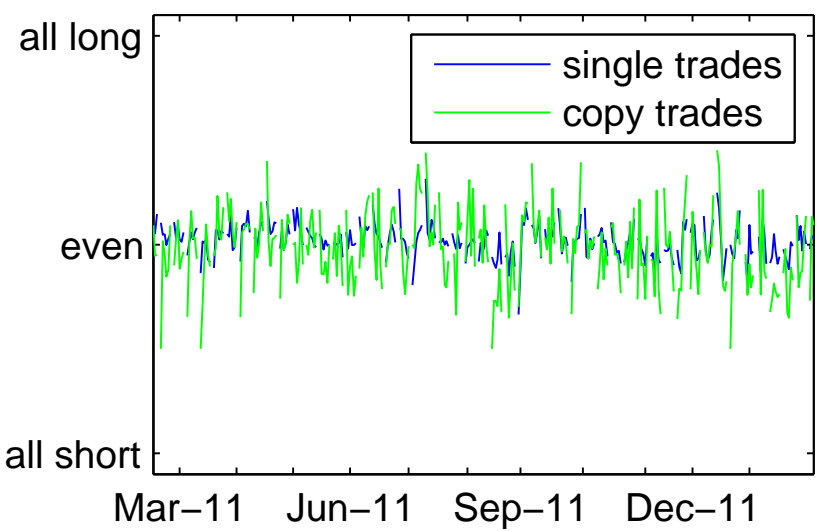

Fig. 6. Daily single market perspective and daily crowd market perspective.

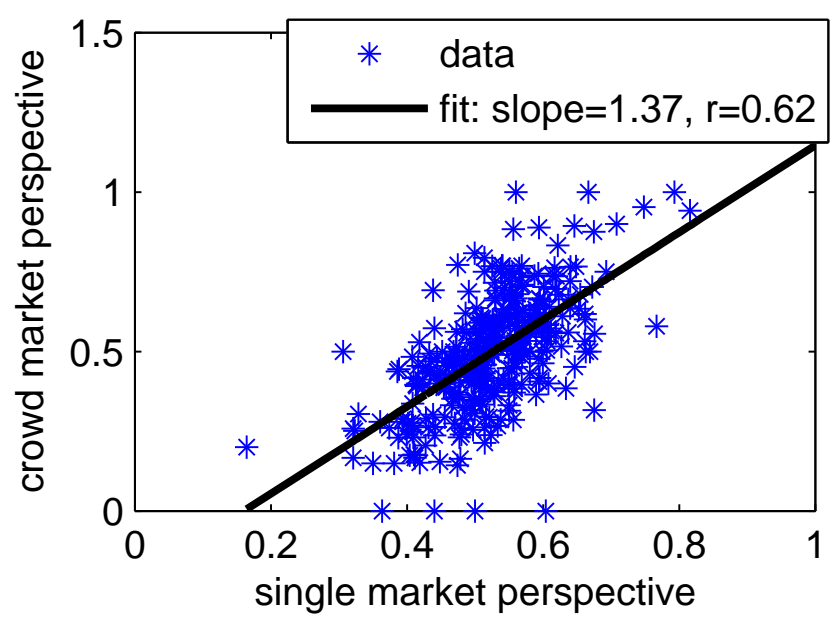

Fig. 7. The single market perspective versus the crowd market perspective for all trading days.

to others' trades drives the market further from parity. For example, when $60 \%$ of the crowd individually decides to short the stock, exposing the crowd to others trading behaviors will likely push $80 \%$ of the crowd to short. If we believe that the average individual opinion of the market is the best price of the market, then social influence seems to encourage overreaction and to drive the market to extreme.

Now we know that social influence has effects on trading behaviors, we continue to investigate that if such influence is consistent all the time. We first define the market trend $t_{d}^{i}$.

$$
t_{d}^{i}=p_{d}-\operatorname{avg}\left(p_{d-i: d}\right),
$$

where $p_{d}$ is today's price, and $\operatorname{avg}\left(p_{i-d: d}\right)$ is the average price from the previous $i$ days, with $i$ as the average window size. The price trend is to eliminate high frequency noise from the market trend. If $t_{d}^{i}$ is positive, the market has a up-going trend; otherwise the market tends to go down.

We correlate both $s_{d}$ and $c_{d}$ with different values of $i \in\{1,3,5,7,14,20\}$, and shift the trend days forward

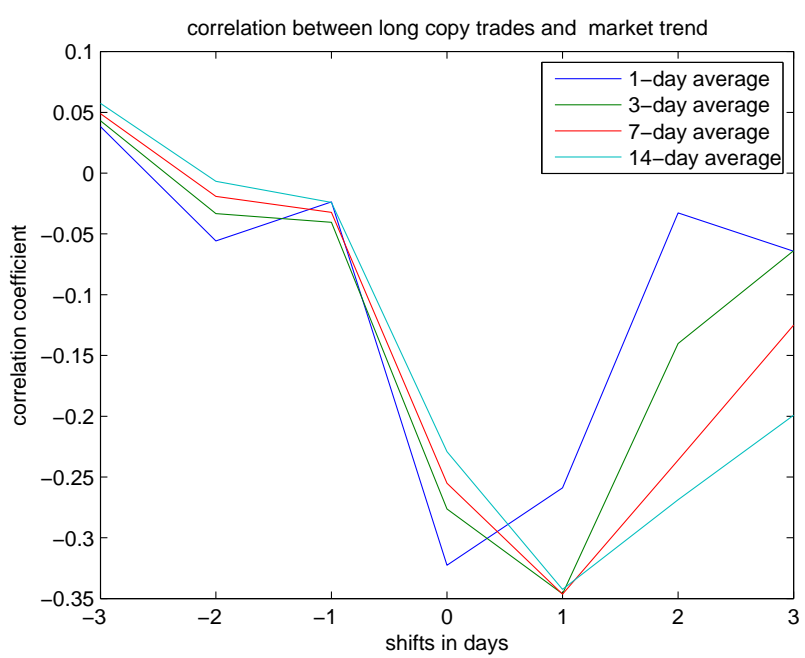

Fig. 8. The correlation coefficient between $c_{d}$ and $t_{d}^{i}$ of different window size $i$ shifted from -3 days to 3 days. The largest correlation occurs when market trend is one day after, with an average window size of 7 and 14 days.

and backward to check if eToro users' market perspective predicts or follows the market trend. We find that there is no significant correlation between market trend and individual $s_{d}(r<0.02, p>0.10)$. However, we observe a strong correlation between the crowd market perspective and the next day's price trend $(r=-0.35, p<0.1)$. The results are shown in Fig. 8 for different window sizes for market trends and different shifts.

We clearly see Fig. 8 that the strongest correlation is at $i \approx 10$ with tomorrow's market trend. As a result, social copy trades can actually be used to predict tomorrow's market trend. Also notice that the coefficient in Fig. 8 is negative, which means that the more long social trades are today (long trades imply expectation of market recovery), the more likely the market is going down tomorrow with respect to its weekly average. We plot both the $s_{d}$ and $t_{d}^{i}$ (shifted by one day) with $i=14$ in Fig. 10, and it is pretty visible to notice that when $s_{d}$ goes extreme in one direction, $t_{d}^{i}$ usually goes the opposite direction.

Surprisingly, such observation connects well with one of the most popular hedge funding trading strategy: the mean reversion strategy [20], which argues that all aggressive market reaction is overreaction, and the market will correct it quickly. The mean reversion strategy then bets on the opposite direction of today's stock trends the next day or so. In our results, the negative correlation suggests that aggressive copy tradings often lead to market overreaction, and the market quickly corrects in the near future.

\section{A. Social Reversion Strategy}

We can use similar ideas from the mean reversion strategy to construct a trading strategy. We balance the portfolio daily. If we notice a day with strongly biased copy trades $\left(c_{d}>0.65\right.$ or $c_{d}<0.35$ ), we will long or short the security with $0.5-c_{d}$ fraction of the total capital we have, which is merely betting 


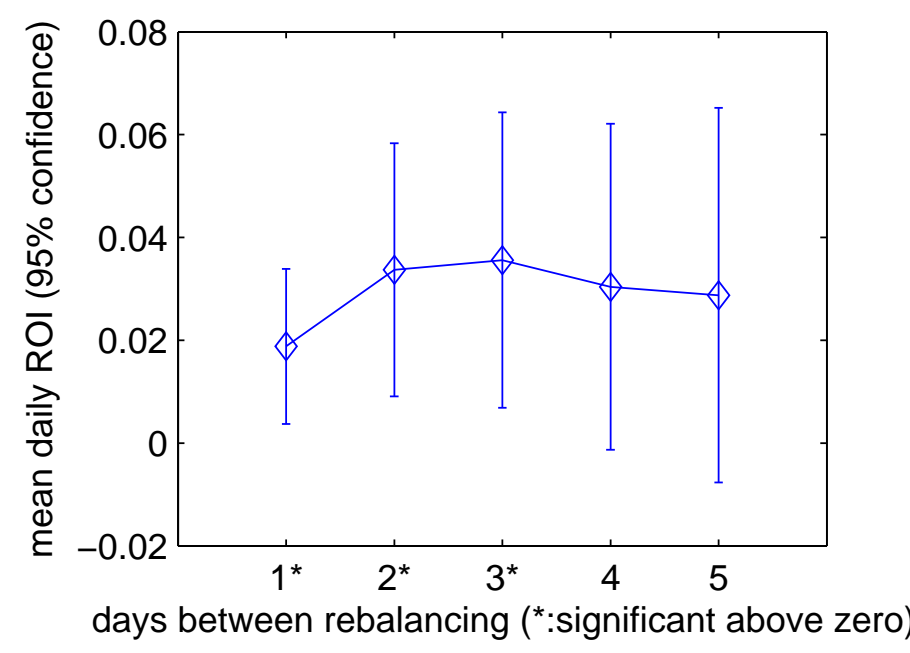

Fig. 9. The mean daily ROI for our mean reversion strategy based on copy trades overreaction. We plot the daily ROI with different holding periods, and we discover that holding 2 to 3 days for market correction provides the best positive return $(*$ : significantly positive with $t$-test $p<0.01$ ).

against the crowd market perspective. The stronger the bias, the more weights we put into our bets. We then hold the position for a certain number of days. Fig. 9 shows the mean ROI for different holding periods, and it seems that waiting 2 or 3 days for market to correct is the most profitable. Since foreign exchange prices move slowly, in this example we increase leverage to $30 \times$.

\section{B. Discussion}

In Fig. 7, we observe that social influence is increasing trading reactions. We argue this is an intuitive observation with the following simple model: Assume a user $i$ at time $d$ is likely to buy an instrument at probability $p$ (denoted as $\operatorname{Prob}\left(X_{i}=1\right)=p$ ) and short an instrument at $1-p$ (denoted as $\operatorname{Prob}\left(X_{i}=0\right)=1-p$ ). If user $i$ consults with another user $j$ sharing a common buy view, the probability of user $i$ buying an instrument will increase by $a, 0 \leq a \ll 1$. Such social influence assumption is based on the common properties of adoption models [18]. Vice versa, if $j$ has a sell view, the probability of user $i$ buying an instrument will be decrease by $a$. Assuming user $j$ has the same distribution $\operatorname{Prob}\left(X_{j}=1\right)=p$, we have:

$$
\begin{aligned}
E\left(X_{i} \text { after consulting } j\right) & \\
= & (p+a) \times \operatorname{Prob}\left(X_{j}=1\right) \\
& +(p-a) \times \operatorname{Prob}\left(X_{j}=0\right) \\
= & (p+a) p+(p-a)(1-p) \\
= & (2 a+1) p-a .
\end{aligned}
$$

With a slope of 1.37 from eToro, we have $a=19 \%$.

With the 2008 economic crisis being still vivid in everyone's mind, understanding the vulnerability of the financial systems becomes one of society's most important problems. Financial researchers have long been aware of the fact of market overreaction and often profit from it [20], and there exist a lot of theories to explain the overreaction [21]. However, eToro data has provided us a unique view on the social influence behavior of traders: The mere fact that a trader looks up on other traders' records will increase trading strategy volatility and market overreaction. We believe that this is an important mechanism for the dynamics of financial systems.

What also surprises us is the result demonstrated in Fig. 8 and Fig. 10. which suggests that social trading can be used as a prediction signal while individual trades can not. We think that each user may have different speculation tendency at different times, and therefore the parameter $a$ is dynamic rather than static. When the market is full of uncertainty and users are more likely to speculate, users are more vulnerable to external influence in decision making processes and tend to overreact. On the other hand, when users are more certain about the market, the external influence plays a less important role in the decision making process. Therefore, the strong signals from social copy trades can be a measure of overreaction as well as market uncertainty, which usually indicates a potential change in market direction.

To better illustrate this point, we also look at the ratio between the daily number of copy trade transactions and the number of single trade transactions. We correlate the ratio of daily single trades over copy trades with the market daily volatility computed using a exponential smoother $\lambda=0.80$ for computing the exponentially weighted volatility risk metric [22] for EUR/USD. The results are shown in Fig. 11. It seems that the two curves correlate positively with $R=0.19, p<0.001$. This implies that users are less likely to be under others' influence and trust more in their own views in the case of strong volatility. Therefore, a very strong piece of information or a clear market trend actually inhibits peer influence and promotes self judgments. As a result, when social influence effects are strong, the market is usually at an uncertain stage, and therefore the crowd market perspective is more overreaction over rational estimations.

\section{CONCLuSion}

We observe the effects of two social mechanisms in the eToro financial system: namely the effects of social feedback in eliciting experts and the effects of social influence when others' trades are available to users. We find that social trading provides much better opportunities for profiting compared with individual trading. We discover that the eToro crowd is making excellent but sometimes not optimal decisions in selecting experts when they can see others' choices. Our finding sheds light on many crowd wisdom systems which expect users to be fully rational under strong financial incentives. We argue that social effects are so strong that they sometimes override the rational assumptions. We also discover that users are prone to much riskier behavior when following their peers, and are much likely to overreact when their peers are doing so and the market is uncertain. This may explain the natural fluctuation of financial markets when there is no external information [23]. 


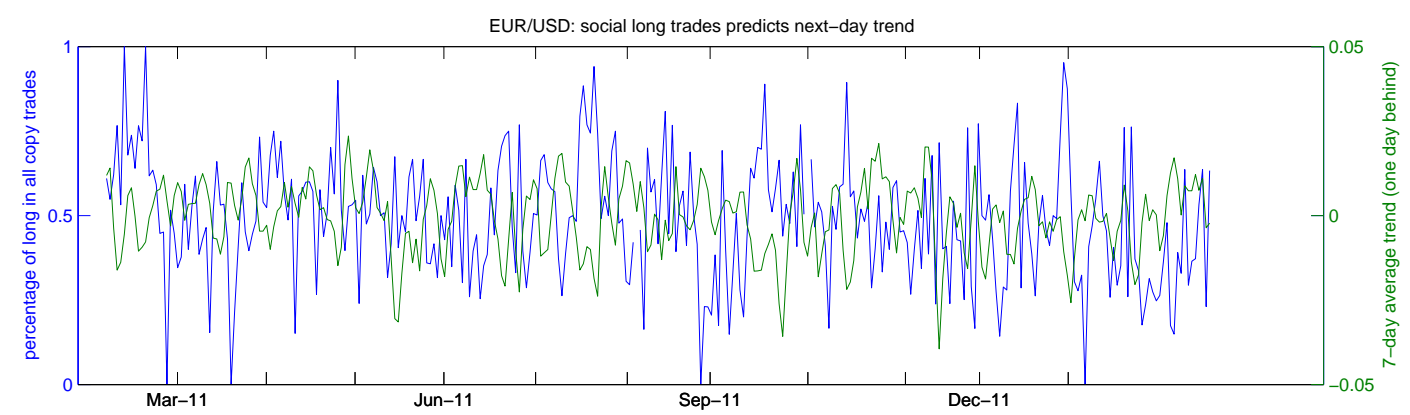

Fig. 10. We plot the crowd market perspective together with the one-day-behind real market trend smoothed by a one-week average window. We notice that the crowd market perspective strongly negatively correlates with the next-day market price.

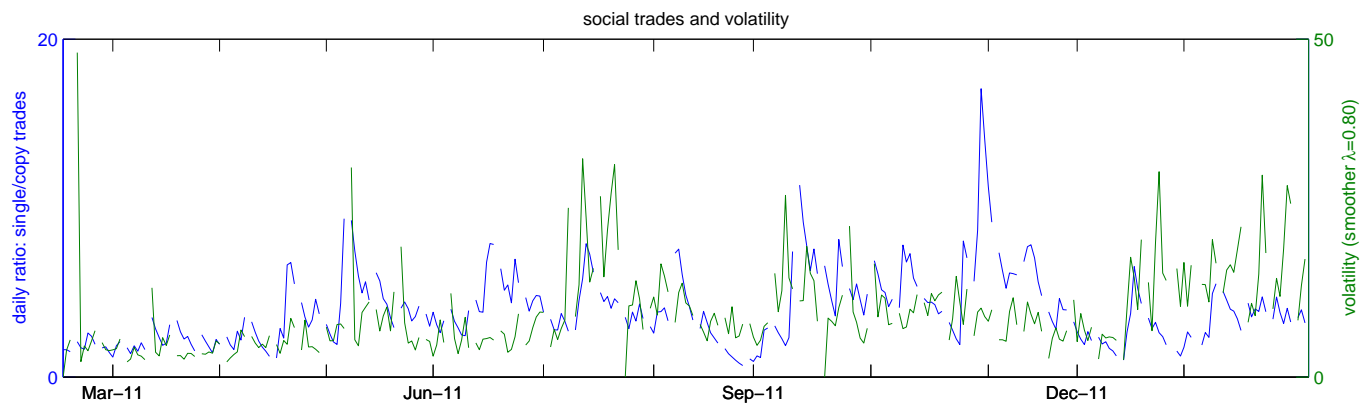

Fig. 11. Volatility of EUR/USD vs. the ratio between single and copy trades.

Future works include a more accurate behavioral model for trading decision making under social influence, and a better way of hedging and portfolio constructions to leverage the potential of crowd wisdom at the same time eliminating the negative effects of social mechanisms on financial systems. Considering the fact that financial systems after all are composed of humans, we believe that this direction of research is potentially valuable.

\section{REFERENCES}

[1] D. Lazer, A. Pentland, L. Adamic, S. Aral, A. Barabasi, D. Brewer, N. Christakis, N. Contractor, J. Fowler, M. Gutmann et al., "computational social science," Science, vol. 323, no. 5915, p. 721, 2009.

[2] A. Woolley, C. Chabris, A. Pentland, N. Hashmi, and T. Malone, "Evidence for a collective intelligence factor in the performance of human groups," science, vol. 330, no. 6004, pp. 686-688, 2010.

[3] N. Aharony, W. Pan, C. Ip, I. Khayal, and A. Pentland, "Social fmri: Investigating and shaping social mechanisms in the real world," Pervasive and Mobile Computing, 2011.

[4] N. Christakis and J. Fowler, "The spread of obesity in a large social network over 32 years," New England Journal of Medicine, vol. 357, no. 4 , pp. $370-379,2007$.

[5] F. Black and M. Scholes, "The pricing of options and corporate liabilities," The journal of political economy, pp. 637-654, 1973.

[6] S. Saavedra, K. Hagerty, and B. Uzzi, "Synchronicity, instant messaging, and performance among financial traders," Proceedings of the National Academy of Sciences, vol. 108, no. 13, p. 5296, 2011.

[7] W. Pan, W. Dong, M. Cebrian, T. Kim, J. Fowler, and A. Pentland, "Modeling dynamical influence in human interaction: Using data to make better inferences about influence within social systems," Signal Processing Magazine, IEEE, vol. 29, no. 2, pp. 77-86, 2012.

[8] M. Billio, L. Pelizzon, A. Lo, and M. Getmansky, "Econometric measures of connectedness and systemic risk in the finance and insurance sectors," 2011.
[9] J. Berg, F. Nelson, and T. Rietz, "Prediction market accuracy in the long run," International Journal of Forecasting, vol. 24, no. 2, pp. 285-300, 2008.

[10] S. Goel, D. Reeves, D. Watts, and D. Pennock, "Prediction without markets," in Proceedings of the 11th ACM conference on Electronic commerce. ACM, 2010, pp. 357-366.

[11] K. Arrow, R. Forsythe, M. Gorham, R. Hahn, R. Hanson, J. Ledyard, S. Levmore, R. Litan, P. Milgrom, F. Nelson et al., "The promise of prediction markets," SCIENCE, vol. 320, no. 5878, p. 877, 2008.

[12] D. Centola and M. Macy, "Complex contagions and the weakness of long ties1," American Journal of Sociology, vol. 113, no. 3, pp. 702734, 2007.

[13] A. Kittur, E. Chi, B. Pendleton, B. Suh, and T. Mytkowicz, "Power of the few vs. wisdom of the crowd: Wikipedia and the rise of the bourgeoisie," World Wide Web, vol. 1, no. 2, p. 19, 2007.

[14] W. Sharpe, "The sharpe ratio," 1994.

[15] M. Salganik, P. Dodds, and D. Watts, "Experimental study of inequality and unpredictability in an artificial cultural market," science, vol. 311, no. 5762 , pp. 854-856, 2006.

[16] A. Barabási and R. Albert, "Emergence of scaling in random networks," science, vol. 286, no. 5439, pp. 509-512, 1999.

[17] A. Pentland and S. Pentland, Honest signals: how they shape our world. The MIT Press, 2008.

[18] W. Pan, N. Aharony, and A. Pentland, "Composite social network for predicting mobile apps installation," in Proceedings of the 25th Conference on Artificial Intelligence, AAAI-11, San Francisco, CA, 2011

[19] Y. Altshuler, W. Pan, and A. Pentland, "Trends prediction using social diffusion models," in International Conference on Social Computing, Behavioral-Cultural Modeling and Prediction, 2012.

[20] A. Lo and A. MacKinlay, "When are contrarian profits due to stock market overreaction?" Review of financial studies, vol. 3, no. 2, pp. 175-205, 1990.

[21] P. Veronesi, "Stock market overreactions to bad news in good times: a rational expectations equilibrium model," Review of Financial Studies, vol. 12, no. 5, pp. 975-1007, 1999.

[22] R. Metrics, "Technical document," JP Morgan, 1997.

[23] P. Koudijs, "The boats that did not sail." 